\title{
Neoadjuvant Stereotactic Body Radiotherapy After Upfront Chemotherapy Improves Pathologic Outcomes Compared With Chemotherapy Alone for Patients With Borderline Resectable or Locally Advanced Pancreatic Adenocarcinoma Without Increasing Perioperative Toxicity
}

Colin S. Hill, MD ${ }^{1}$, Lauren M. Rosati, MD ${ }^{2}$, Chen Hu, PhD, MS ${ }^{1}$, Wei Fu, PhD $^{1}$, Shuchi Sehgal, BA ${ }^{3}$, Amy Hacker-Prietz, PA-C ${ }^{1}$, Christopher L. Wolfgang, MD, $\mathrm{PhD}^{4}$, Matthew J. Weiss, $\mathrm{MD}^{\mathbf{5}}$, Richard A. Burkhart, $\mathrm{MD}^{6}$, Ralph H. Hruban, $\mathrm{MD}^{7}$, Ana De Jesus-Acosta, $\mathrm{MD}^{8}$, Dung T. Le, $\mathrm{MD}^{8}$, Lei Zheng, MD, $\mathrm{PhD}^{8}$, Daniel A. Laheru, $\mathrm{MD}^{8}$, Jin $\mathrm{He}, \mathrm{MD}, \mathrm{PhD}^{4}$, Amol K. Narang, $\mathrm{MD}^{1}$, and Joseph M. Herman, MD, MSc, MSCHM ${ }^{9}$

${ }^{1}$ Department of Radiation Oncology and Molecular Radiation Sciences, Johns Hopkins University School of Medicine, Sidney Kimmel Cancer Center, Baltimore, MD; ${ }^{2}$ University of South Carolina School of Medicine, Columbia, SC; ${ }^{3}$ Philadelphia College of Osteopathic Medicine, Philadelphia, PA; ${ }^{4}$ Department of Surgery, New York University Grossman School of Medicine, New York, NY; ${ }^{5}$ Department of Surgery, Zucker School of Medicine at Hofstra/Northwell, Lake Success, NY; ${ }^{6}$ Department of Surgery, Johns Hopkins University School of Medicine, Baltimore, MD; ${ }^{7}$ Department of Pathology, the Sol Goldman Pancreatic Cancer Research Center, Johns Hopkins University School of Medicine, Baltimore, MD; ${ }^{8}$ Department of Oncology, Johns Hopkins University School of Medicine, Baltimore, MD; ${ }^{9}$ Radiation Medicine, Zucker School of Medicine at Hofstra/Northwell, Lake Success, NY

\begin{abstract}
Background. Patients with borderline resectable pancreatic cancer (BRPC) or locally advanced pancreatic cancer (LAPC) are at high risk of margin-positive resection. Neoadjuvant stereotactic body radiation therapy (SBRT) may help sterilize margins, but its additive benefit beyond neoadjuvant chemotherapy (nCT) is unclear. The authors report long-term outcomes for BRPC/LAPC
\end{abstract}

Colin S. Hill and Lauren M. Rosati should be considered joint first authors.

(C) The Author(s) 2022

First Received: 22 April 2021

Accepted: 15 November 2021;

Published Online: 7 February 2022

A. K. Narang, MD

e-mail: Anarang2@jhmi.edu

J. M. Herman, MD, MSc, MSCHM

e-mail: Jherman1@ northwell.edu patients explored after treatment with either $\mathrm{nCT}$ alone or nCT followed by five-fraction SBRT (nCT-SBRT).

Methods. Patients with BRPC or LAPC from 2011 to 2016 who underwent resection after nCT alone or nCTSBRT were retrospectively reviewed. Baseline characteristics were compared, and the propensity score with inverse probability weighting (IPW) was used to compare pathologic/survival outcomes.

Results. Of 198 patients, 76 received nCT, and 122 received nCT-SBRT. The nCT-SBRT cohort had a higher proportion of LAPC (53\% vs $22 \%$; $p<0.001$ ). The duration of nCT was longer for nCT-SBRT (4.6 vs 2.9 months; $p=0.03)$, but adjuvant chemotherapy was less frequently administered (53\% vs $67.1 \% ; p<0.001)$. Adjuvant radiation was administered to $30 \%$ of the nCT patients. The nCT-SBRT regimen more frequently achieved negative margins ( $92 \%$ vs $70 \% ; p<0.001)$, negative nodes $(59 \%$ vs $42 \% ; p<0.001)$, and pathologic complete response (7\% vs $0 \% ; p=0.02)$. In the multivariate analysis, nCT-SBRT remained associated with $\mathrm{R} 0$ resection $(p<0.001)$. The nCT-SBRT cohort experienced no significant difference in median overall survival (OS) (22.1 vs 24.5 months), local 
progression-free survival (LPFS) (13.5 vs. 15.4 months), or distant metastasis-free survival (DMFS) (11.7 vs 16.3 months) after surgery. After SBRT, 1-year OS was 77.0\% and 2-year OS was $50.4 \%$. Perioperative Claven-Dindo grade 3 or greater morbidity did not differ significantly between the nCT and nCT-SBRT cohorts $(p=0.81)$.

Conclusions. Despite having more advanced disease, the nCT-SBRT cohort was still more likely to undergo an R0 resection and experienced similar survival outcomes compared with the $\mathrm{nCT}$ alone cohort.

Pancreatic adenocarcinoma remains an aggressive malignancy with dismal long-term survival outcomes and is expected to become the second leading cause of cancer death by the year 2030. ${ }^{1,2}$ Outcomes are poor, as roughly half of patients present with evidence of metastatic disease at diagnosis. The majority of patients without metastatic disease present with extra-pancreatic extension and involvement of key peri-pancreatic vasculature, rendering complete surgical resection with negative margins challenging.

Nevertheless, response rates for nonoperative therapies have improved, enabling a higher proportion of patients with localized disease to undergo complete resection compared with historical data. ${ }^{3-8}$ Indeed, multi-agent systemic regimens, such as FOLFIRINOX (FFX) and gemcitabine combined with nab-paclitaxel (GnP), which were first demonstrated to improve survival in the metastatic setting, have since been administered in the localized setting with encouraging outcomes. ${ }^{9-11}$

Additionally, advances in radiation technologies have dramatically improved the precision of treatment delivery for pancreatic tumors. One example is the use of the hypofractionated stereotactic body radiation therapy (SBRT), in which precisely delivered high doses of radiation per fraction allows treatment during a much shorter interval, usually five fractions or less. ${ }^{12,13}$

In the setting of candidacy for surgical exploration, neoadjuvant radiation can be administered with the goal of increasing the likelihood of margin-negative resection and decreasing the risk of postoperative local recurrence. Either chemoradiation (CRT) or SBRT can be used in this setting, but the latter has value in minimizing the interval between the end of chemotherapy and surgical exploration and in reducing the risk of acute toxicity, treatment-related lymphopenia, and quality-of-life decrement. ${ }^{14-16}$

Nevertheless, the additive benefit of radiation beyond neoadjuvant chemotherapy alone for patients with borderline resectable pancreatic cancer (BRPC) or locally advanced pancreatic cancer (LAPC) undergoing surgical exploration has not been well-characterized to date because existing data are limited by the lack of inclusion of modern multi-agent systemic regimens and radiation technology. ${ }^{17-19}$ This report describes the long-term pathologic and survival outcomes in addition to the 90-day perioperative toxicity for a cohort of patients treated at single highvolume institution who underwent subsequent surgical resection after receiving neoadjuvant chemotherapy (nCT) alone or neoadjuvant chemotherapy followed by SBRT (nCT-SBRT).

\section{METHODS}

Patient Population and Treatment Course: Neoadjuvant Chemotherapy and SBRT

With institutional review board approval, all patients with BRPC and LAPC diagnosed between 2011 and 2016 at our institution who were subsequently explored for surgical resection after $\mathrm{nCT}$ or sequential nCT-SBRT were retrospectively reviewed. Patient eligibility and follow-up evaluation are discussed in Appendix 1. Systemic therapy was prescribed at the discretion of the treating medical oncologist. Robust patients with good performance status generally received induction multi-agent chemotherapy such as modified FOLFIRINOX (mFFX) or non-modified FFX, GnP, or gemcitabine, docetaxel, and capecitabine (GTX).

During induction therapy, the patients were serially examined at 2- to 3-month intervals with a pancreatic protocol computed tomography scan to assess chemo-responsiveness and confirm continued treatment eligibility. Although standard practice was to offer SBRT after chemotherapy, if patients had a robust response to chemotherapy with limited vessel involvement, they sometimes underwent surgery without SBRT. For those who received nCT-SBRT, our institutional practice regarding SBRT has previously been described in detail (Appendix 2). ${ }^{12}$

\section{Clinical Characteristics and Treatment Outcomes}

Chemotherapy received after SBRT and before surgery in the nCT-SBRT cohort was included in the calculation of neoadjuvant CT duration. After surgery, pathologic outcomes were centrally reviewed for tumor grade, margin status, and nodal clearance by two dedicated pathologists who re-reviewed the specimens. Pathologic complete response (pCR), was defined as no residual viable tumor, whereas near pCR (npCR) was defined as single cells or rare small groups of cancer cells. ${ }^{20}$ Partial response was defined as residual cancer with evident regression but more than single cells or rare small groups of cancer cells. ${ }^{20}$ 
Absent response was assigned when extensive cancer was present with no evident tumor regression. ${ }^{20}$

Perioperative toxicity was collected for 90 days after surgery, and complications were graded according to the Claven-Dindo classification. ${ }^{21}$ Perioperative toxicity was compared between cohorts with the chi-square test or Fisher's exact test. Statistical analysis of survival measures, including overall survival (OS), progression freesurvival (PFS), local PFS (LPFS), and distant metastasisfree survival (DMFS) and of how covariates and outcomes were compared by cohort are summarized in Appendix 3.

\section{RESULTS}

\section{Clinical Demographics and Treatment Characteristics}

Retrospective review identified 198 consecutive patients with BRPC or LAPC who met our inclusion criteria. The baseline clinical demographics are summarized in Table 1. Of the 198 patients, $76(38.4 \%)$ received $\mathrm{nCT}$ and 122 $(61.6 \%)$ received nCT-SBRT. No significant difference in age, gender, performance status, tumor location, or CA 19-9 level was observed at diagnosis or post-nCT (all $p>0.05)$. However, the nCT-SBRT cohort had a significantly higher percentage of LAPC patients $(53.3 \%$ vs $22.4 \% ; p<0.05)$.

Regarding chemotherapy type, mFFX was the most commonly administered chemotherapy regimen in both cohorts $(56.6 \%$ nCT vs $54.5 \%$ nCT-SBRT), whereas GNP was the second regimen of choice $(14.5 \%$ nCT vs. $15.6 \%$ nCT-SBRT). The type of chemotherapy administered by cohort did not differ significantly, but the total induction chemotherapy duration differed significantly between the nCT and nCT-SBRT cohorts (2.9 vs 4.6 months; $p=0.029)$. In the nCT-SBRT cohort, 74 patients $(62.2 \%)$ received more than 4 months of induction chemotherapy compared with 23 patients $(31.1 \%)$ in the nCT cohort $(p<0.001)$.

Regarding adjuvant therapy, systemic chemotherapy was more frequently administered after surgery in the nCT cohort $(67.1 \%$ vs $52.5 \% ; p<0.001)$, and for longer durations (4.0 vs 2.1 months; $p=0.003$ ). Notably, 23 patients $(30.3 \%)$ in the $\mathrm{nCT}$ cohort also received adjuvant radiation after surgery for high-risk features, namely, margin positivity in 14 patients $(61 \%)$ and/or node-positive resections in 20 patients $(87 \%)$.

\section{Pathologic Outcomes After Neoadjuvant Therapy}

Pathologic outcomes were significantly better in the nCT-SBRT cohort than in the nCT-alone cohort (Table 2). Margin sterilization was achieved for 112 patients $(91.8 \%)$ in the nCT-SBRT cohort compared with only 53 patients $(69.7 \%)$ in the nCT cohort $(\mathrm{p}<0.001)$. Only 32 patients $(42.1 \%)$ were pathologically node-negative in the nCT cohort compared with 72 patients $(59.0 \%)$ in the nCTSBRT cohort $(p=0.028)$. Notably, none of the patients in the nCT-alone cohort achieved a pCR, but nine patients $(7.4 \%)$ in the nCT-SBRT cohort achieved a pCR $(p=0.001)$. The multivariate analysis with propensity score analysis based on IPTW using the propensity score demonstrated that receipt of SBRT still was significantly associated with negative margins, but not for nodal clearance after accounting for potential confounding (Table 3). Vasculature reconstruction was less frequently required in the nCT cohort (20 patients [26.3\%]: 18 BRPC patients [30.5\%] and 2 LAPC patients [11.8\%]). In the nCT-SBRT cohort, 49 patients $(40.2 \%)$ required reconstruction, including 23 BRPC patients (40.3\%) and 26 LAPC patients $(40.0 \%)$. Among the nCT patients, the median fibrosis of the resected tumor was 35\% (range, 0-99\%) compared with $70 \%$ (range, 0-100\%) among the nCT-SBRT cohort.

\section{Survival Outcomes and Patterns of Failure}

Kaplan-Meier analysis showed no significant differences in survival outcomes based on induction therapy type (Table 4; Fig. 1). The median OS after surgery was 22.1 months (range, 17.6-29.3 months) in the nCT-SBRT cohort compared with 24.5 months (range, 18.6-31.6 months) in the CT cohort $(p>0.05)$. After SBRT, the 1-year OS probability was $77.0 \%$ (95\% confidence interval [CI], 69.9-84.9\%) and the 2-year OS probability was $50.4 \%$ (95\% CI, 42.0-60.2\%) for the nCT-SBRT cohort. The LAPC patients treated with nCT-SBRT trended toward a better OS than the patients treated with nCT alone ( $p>0.05$; Fig. 2). In the nCT cohort, 28 patients $(36.8 \%)$ were still alive 36 months after diagnosis compared with 47 patients $(38.5 \%)$ in the nCT-SBRT cohort, with 3-year OS probabilities of $31.4 \%$ (95\% CI, 20.6-42.2\%) and $35.9 \%$ (95\% CI, 27.3\%-44.4\%), respectively ( $p>0.05)$.

The median PFS was 13.0 months (range, 10.1-17.7 months) in the nCT cohort versus 11.0 months (range, 8.9-12.4 months) in the nCT-SBRT cohort ( $p>0.05)$. The patterns of failure did not differ significantly between the two groups ( $p=0.306$; Table 4 ). The site of first failure was local in $27 \%$ of the nCT cohort versus $21 \%$ of the nCT-SBRT cohort. Distant first failure occurred in $23 \%$ of the nCT cohort versus $30 \%$ of the nCT-SBRT cohort. Synchronous failure occurred for $14 \%$ of the nCT cohort versus $22 \%$ of the nCT-SBRT cohort. No significant differences in LPFS (15.4 vs 13.5 months; $p>0.05)$ or DMFS (15.4 vs 11.7 months; $p=0.335$ ) were observed.

Multivariable analysis with IPTW demonstrated no significant differences in OS, PFS, LPFS, or DMFS by 
TABLE 1 Clinical demographics

\begin{tabular}{|c|c|c|c|}
\hline & $\begin{array}{l}\text { Chemotherapy } \\
n(\%)\end{array}$ & $\begin{array}{l}\text { Chemotherapy and SBRT } \\
n(\%)\end{array}$ & $p$ Value $<0.05$ \\
\hline$n$ & 76 & 122 & \\
\hline Median age: years (range) & $63.9(40.3-83.2)$ & $63.5(39.7-83.6)$ & \\
\hline \multicolumn{4}{|l|}{ Gender } \\
\hline Female & $33(43.4)$ & $58(47.5)$ & \\
\hline Male & $43(56.6)$ & $64(52.5)$ & \\
\hline \multicolumn{4}{|l|}{ ECOG PS } \\
\hline 0 & $22(29.0)$ & $48(39.3)$ & \\
\hline $1-2$ & $27(35.5)$ & $73(59.8)$ & \\
\hline Not reported & $27(35.5)$ & $1(0.8)$ & \\
\hline \multicolumn{4}{|l|}{ Tumor location } \\
\hline Head/neck/uncinate & $59(77.6)$ & $82(67.2)$ & \\
\hline Body/tail & $17(22.4)$ & $40(32.8)$ & \\
\hline NCCN staging & & & $<0.001$ \\
\hline BRPC & $59(77.6)$ & $57(46.7)$ & \\
\hline LAPC & $17(22.4)$ & $65(53.3)$ & \\
\hline \multicolumn{4}{|l|}{ Median CA 19-9 level (range) } \\
\hline Baseline & $122.5(9.2-1552.6)$ & $190.8(<1.0-14,004.2)$ & \\
\hline After nCT & $54.4(<1.0-913)$ & $32.5(<1.0-2851.4)$ & \\
\hline \multicolumn{4}{|l|}{ CT agent } \\
\hline FFX & $43(56.6)$ & $66(54.1)$ & \\
\hline $\mathrm{GnP}$ & $11(14.5)$ & 19 (15.6) & \\
\hline Other & $22(28.9)$ & $37(30.3)$ & \\
\hline \multicolumn{4}{|c|}{ Median induction CT duration: months (range) } \\
\hline Total duration: months (range). & $2.9(0.7-16.8)$ & $4.6(0-16.5)$ & 0.029 \\
\hline$\geq 4$ Months duration & $23(31.1)$ & $74(62.2)$ & $<0.001$ \\
\hline \multicolumn{4}{|l|}{ Adjuvant therapy } \\
\hline $\mathrm{CT}$ & $51(67.1)$ & $64(52.5)$ & 0.001 \\
\hline Median CT duration: months (range) & $4.0(0-18.5)$ & $2.1(0-6.0)$ & 0.003 \\
\hline Radiation & $23(30.3)$ & N/A & N/A \\
\hline
\end{tabular}

SBRT, stereotactic body radiation therapy; ECOG PS, Easteron Cooperative Oncology Group performance status; NCCN, National Comprehensive Cancer Network; BRPC, borderline resectable pancreatic adenocarcinoma; LAPC, locally advanced pancreatic adenocarcinoma; CA 19-9, cancer antigen 19-9; FFX, FOLFIRINOX; GnP, gemcitabine and nab-paclitaxel; CT, chemotherapy

cohort (Table 3). However, when the patients surviving longer than 36 months were compared with those who had a shorter survival, the patients who survived less than 36 months more frequently had positive margins $(37.5 \%$ vs $17.9 \%$ in $\mathrm{nCT}$ [ $p=0.037] ; 12.0 \%$ vs. $2.1 \%$ in $\mathrm{nCT}-\mathrm{SBRT}$ $[p=0.025])$ as well as nodal positivity (67\% vs $39 \%$ in nCT $[p=0.01] ; \quad 50.7 \%$ vs $27.7 \%$ in nCT-SBRT $[p=0.03])$, and the differences were significant. No patients in the nCT cohort achieved a pCR, but this was significantly associated with long-term survivors in the nCT-SBRT cohort $(4.0 \%$ in $<36$ months vs $12.8 \%$ in $\geq 36$ months; $p=0.039$ ). Survival outcomes based on margins, nodal status, and pCR are demonstrated in Fig. S1A-C.

\section{Perioperative Morbidity}

Perioperative toxicity was available for $72 \mathrm{nCT}$ patients and $122 \mathrm{nCT}$-SBRT patients after surgery. The readmission rates and 90-day post-surgery mortality rates did not differ significantly between the cohorts, as demonstrated in Table 5.

Complications were graded according to the ClavienDindo classification, and the rate of grade 3 or greater toxicity did not differ significantly between the cohorts, with 11 events $(15.3 \%)$ in the nCT cohort versus 15 events $(12.3 \%)$ in the nCT-SBRT cohort $(p=0.81)$. Bleeding events occurred for two patients in the nCT cohort, with one patient requiring an embolization for a bleed from the 
TABLE 2 Pathologic outcomes

\begin{tabular}{llll}
\hline & $\begin{array}{l}\text { Chemotherapy } \\
(n=76) \\
n(\%)\end{array}$ & $\begin{array}{l}\text { Chemotherapy and SBRT } \\
(n=122) \\
n(\%)\end{array}$ & $p$ Value $<0.05$ \\
& $53(69.7)$ & $112(91.8)$ & 0.0001 \\
\hline $\begin{array}{l}\text { Margin status } \\
\text { Negative }\end{array}$ & $23(30.3)$ & $10(8.2)$ & 0.0279 \\
$\begin{array}{l}\text { Positive } \\
\text { Nodal status }\end{array}$ & $32(42.1)$ & $72(59.0)$ & \\
Node-negative & $44(57.9)$ & $50(41.0)$ & 0.001 \\
Node-positive & & $9(7.4)$ & \\
Pathologic response & $0(0.0)$ & $38(31.1)$ & \\
Complete response & $12(15.8)$ & $75(61.5)$ & \\
Nearly complete response & $64(84.2)$ & & \\
Other & &
\end{tabular}

SBRT, stereotactic body radiation therapy
TABLE 3 Propensity score-adjusted multivariable analysis

\begin{tabular}{|c|c|c|c|}
\hline & OR & $95 \% \mathrm{CI}$ & $p$ Value $<0.05$ \\
\hline \multicolumn{4}{|c|}{ Surgical margin } \\
\hline ATT & 0.82 & $0.69-0.97$ & 0.0203 \\
\hline ATE & 0.82 & $0.72-0.94$ & 0.004 \\
\hline \multicolumn{4}{|c|}{ Pathologically nodal-negative } \\
\hline ATT & 0.93 & $0.76-1.13$ & 0.4487 \\
\hline ATE & 0.92 & $0.78-1.09$ & 0.3403 \\
\hline \multicolumn{4}{|c|}{ Overall survival } \\
\hline ATT & 0.96 & $0.62-1.50$ & 0.8741 \\
\hline ATE & 0.95 & $0.66-1.38$ & 0.7977 \\
\hline \multicolumn{4}{|c|}{ Progression-free survival } \\
\hline ATT & 1.04 & $0.72-1.51$ & 0.8269 \\
\hline ATE & 1.1 & $0.79-1.54$ & 0.5682 \\
\hline \multicolumn{4}{|c|}{ Local progression-free survival } \\
\hline ATT & 0.8 & $0.55-1.16$ & 0.2332 \\
\hline ATE & 0.84 & $0.59-1.19$ & 0.322 \\
\hline \multicolumn{4}{|c|}{ Distant metastasis-free survival } \\
\hline ATT & 1.23 & $0.84-1.79$ & 0.2843 \\
\hline ATE & 1.26 & $0.90-1.77$ & 0.185 \\
\hline
\end{tabular}

$\overline{\mathrm{OR} \text {, odds ratio; CI, confidence interval; ATT, after-treatment effect }}$ on the treated; ATE, average treatment effect

right hepatic artery (RHA) and another patient with a gastroduodenal artery (GDA) bleed that was conservatively managed. In the nCT-SBRT cohort, bleeding events occurred for seven patients, including four patients who underwent embolization for bleeds from the GDA, RHA, common hepatic artery, and celiac artery, respectively. A fifth patient underwent placement of a stent for a GDA bleed. An additional patient experienced a bleed from the cystic plate, but this event was thought to be related to an irreversible electroporation procedure and was managed conservatively. An additional patient was admitted for a bleed from an upper gastrointestinal source at an outside hospital, but detailed records for this event with respect to the source and type of interventions received were not available.

\section{DISCUSSION}

In a large cohort of BRPC and LAPC patients treated at a high-volume institution, neoadjuvant CT-SBRT significantly improved pathologic outcomes $(>20 \%$ increase in the margin-negative resection rate) compared with neoadjuvant chemotherapy alone. Furthermore, the rate of grade 3 or greater complications according to the Clavien-Dindo classification did not differ significantly between the cohorts. Bleeding events did not differ significantly between the cohorts, although a marginal increase in the SBRT cohort cannot be ruled out and warrants further study. Despite the significantly higher percentage of LAPC patients in the nCT-SBRT cohort, survival outcomes did not differ between the two cohorts from the date of surgery.

The role of radiation for patients with localized pancreatic cancer remains controversial. In the locally advanced setting, several historical randomized controlled trials (RCTs) have shown mixed results with the addition of either upfront or consolidative radiation to chemotherapy, but the antiquated techniques and chemotherapeutic agents used in these studies render their applicability to modern day practice questionable. ${ }^{22-25}$

The most modern RCT was LAP07, in which LAPC patients were treated with upfront gemcitabine for four cycles and those without progression were subsequently randomized to two additional cycles of gemcitabine or consolidative CRT. ${ }^{19}$ Although LAP07 was negative for 
TABLE 4 Survival and failure outcomes

\begin{tabular}{lll}
\hline & Chemotherapy & Chemotherapy and SBRT \\
\hline Median survival outcomes: months $(95 \%$ CI) & & \\
OS & $24.5(18.6-31.6)$ & $22.1(17.6-29.2)$ \\
PFS & $13.0(10.1-17.7)$ & $11.0(8.9-12.4)$ \\
LPFS & $15.4(11.3-19.4)$ & $13.5(11.3-16.7)$ \\
DMFS & $16.3(11.4-28.2)$ & $11.7(9.4-15.9)$ \\
Failure patterns: $n(\%)$ & & \\
Patients with imaging follow-up after surgery $(n)$ & 64 & 119 \\
Local recurrence & $17(26.6)$ & $25(21)$ \\
Distant recurrence & $15(23.4)$ & $36(30.3)$ \\
Local and distant recurrence & $9(14.1)$ & $26(21.8)$ \\
\hline
\end{tabular}

SBRT, stereotactic body radiation therapy; CI, confidence interval; OS, overall survival; PFS, progression-free survival; LPFS, local progressionfree survival; DMFS, distant metastasis-free survival

the primary end point of OS, the patients in the CRT cohort did experience significantly improved local control $(32 \%$ vs $46 \%) .{ }^{19}$ Given that systemic therapy was gemcitabine alone, systemic control was poor, with $60 \%$ of the patients eligible for randomization to CRT due to progression. ${ }^{19}$ Furthermore, less than $5 \%$ of the patients in the study were surgically explored, which prevented the ability to assess the role of radiation for margin sterilization and local recurrence risk reduction in LAPC. ${ }^{19}$

Since LAP07, several reports from high-volume institutions, including ours, have shown much higher rates of resection and increased OS in the setting of multi-agent chemotherapy regimens such as FOLFIRINOX. ${ }^{3-5,26-29}$ Notably, these reports describe patients who also have been treated nearly universally with neoadjuvant radiation therapy. High rates of margin sterilization in the locally advanced setting after chemotherapy alone has yet to be demonstrated, but the inferior margin-negative resection rate of $69.7 \%$ suggests the added value of radiation in this context. ${ }^{4,26-29}$

Newer studies recently in which patients received FFX followed by CRT have reported favorable R0 resection rates, ranging from $88 \%$ to $100 \%$. $^{3,30-32}$ One study compared FFX followed by CRT or chemotherapy alone, and as in our study, outcomes were improved with the addition of CRT. ${ }^{33}$ A single-arm study of 50 patients in Italy with neoadjuvant FFX and SBRT reported that SBRT delivery to the 39 patients without progression after FFX was associated with a significantly improved median OS (18 months in the SBRT group vs 5 months in the non-SBRT group). ${ }^{34}$ After SBRT, seven patients had an RO resection and experienced a significantly better 3-year OS than nonresected patients $(43 \%$ vs $6.5 \%){ }^{34}$
In a recent phase 1 trial in the Netherlands, 50 patients were treated with eight cycles of FFX followed by SBRT. ${ }^{35}$ Due to progression or toxicity with FFX, only $78 \%$ of the patients proceeded to receive SBRT. Six patients who underwent a resection after FFX or SBRT had R0 resection with a complete $\mathrm{pCR} .{ }^{34}$ The median OS for these six patients after resection was 23 months (95\% CI, 13-34 months). ${ }^{35}$

Reports on the use of CRT in the borderline resectable setting have demonstrated improved pathologic outcomes and in some studies improved OS that mimics resectable disease in those able to undergo subsequent surgery. Although the patient numbers were small, an RCT from Korea exploring upfront CRT versus upfront surgery in BRPC was terminated early due to a much higher rate of margin sterilization in the CRT cohort. ${ }^{17}$ Similarly, the PREOPANC study randomized both resectable and BRPC patients to upfront surgery versus gemcitabine-based CRT. ${ }^{18}$ Although the study did not show a difference for its primary endpoint of OS for the entire cohort with the use of neoadjuvant chemoradiation, a dramatic difference was seen in margin sterilization favoring the CRT cohort $(70 \%$ vs $40 \% ; p<0.001)$, and more importantly, a significant improvement was seen in the LPFS and PFS intervals. Furthermore, in the subset of BRPC patients, OS was improved in the CRT cohort compared with upfront surgery.

Of course, these results raise the question concerning the value of preoperative radiation in the setting of neoadjuvant multi-agent systemic therapy, which was explored in two studies led by the Alliance consortium. Specifically, Alliance A021101 was a single-arm study evaluating FFX followed by CRT for BRPC patients. The median OS in this study was 21.7 months, and $15(68 \%)$ of 22 patients underwent resection with a margin-negative resection rate 


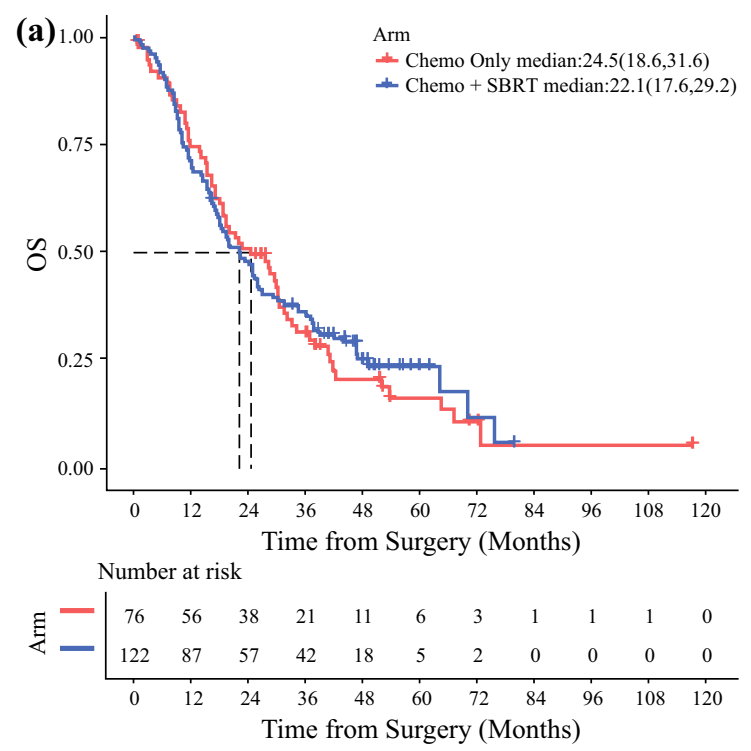

(c)

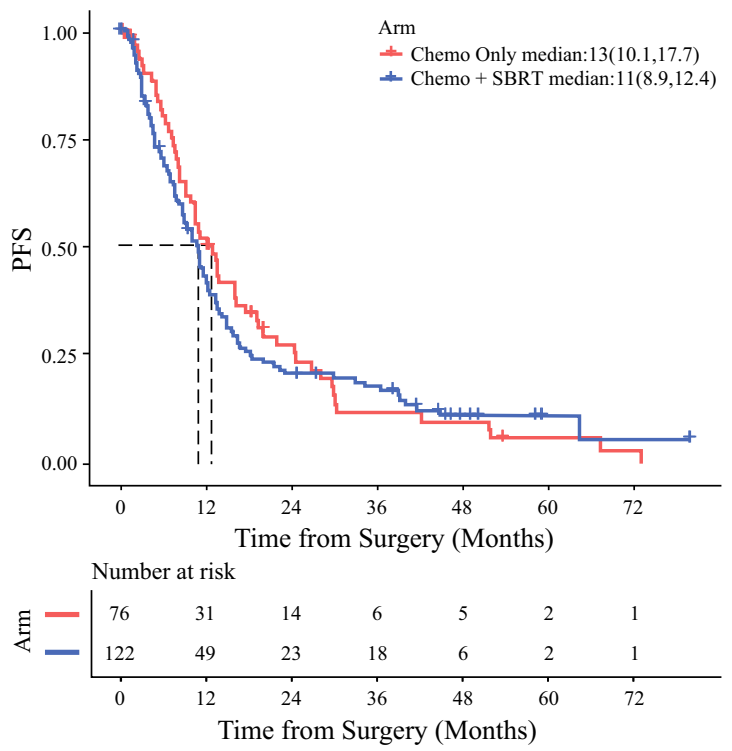

FIG. 1 Kaplan-Meier curves of A overall survival (OS), B progression-free survival (PFS), C local progression-free survival (LPFS), and D distant metastasis-free survival (DMFS) for the

of $93 \% .^{36}$ The resected patients experienced a significantly better 18 -month OS than the non-resected patients $(67 \%$ vs $43 \%) .{ }^{36}$ Importantly, all the patients were treated at highvolume pancreatic centers, and $73 \%$ required vessel reconstruction.

A subsequent study, Alliance 021501, was designed in a randomized fashion to explore the additive value of radiation beyond neoadjuvant FFX alone. ${ }^{37}$ In this study, hypofractionated radiation therapy (SBRT, 40 Gy in 5 fractions or $25 \mathrm{~Gy}$ in 5 fractions if constraints were not made due to anatomy) was administered. A higher proportion of patients in the SBRT cohort failed to undergo R0 resections, resulting in inferior OS. ${ }^{37}$ Although the exact

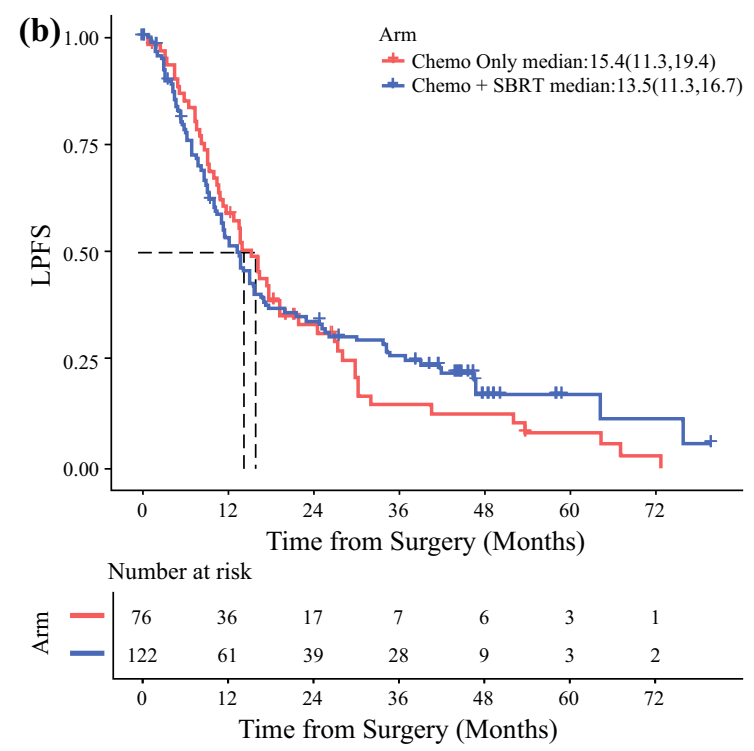

(d)

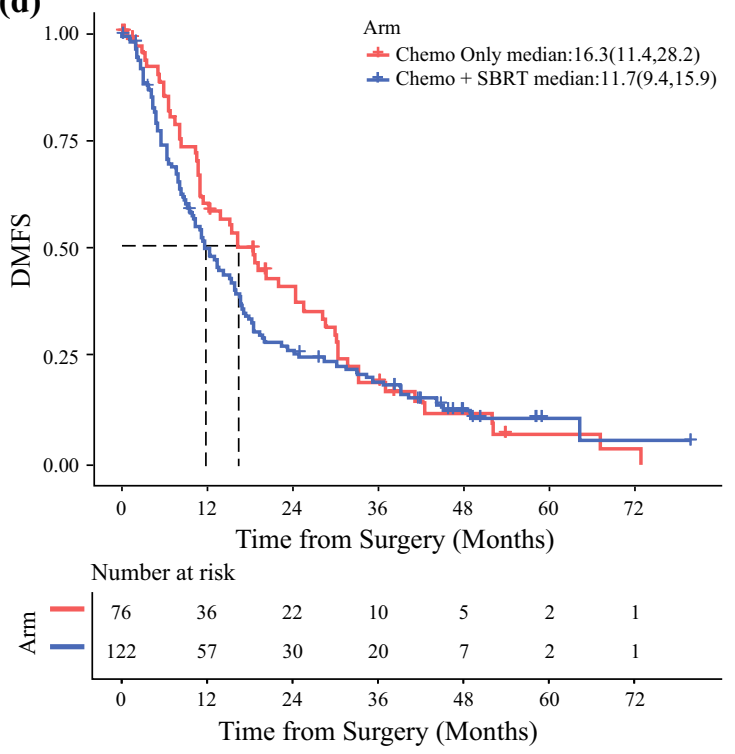

neoadjuvant chemotherapy (nCT) and nCT-SBRT cohorts from the date of surgery. SBRT, stereotactic body radiation therapy

cause was unclear, the SBRT cohort had significantly higher CA 19-9 markers at baseline, had metastases develop in a higher proportion of patients before or at the time of surgery, and had a higher proportion of patients who did not undergo surgery despite localized disease likely due to acute inflammation attributed to radiation therapy. Because only 40 patients in this study received SBRT, it was not powered to definitively determine the role of SBRT in this setting, but it did suggest that the addition of SBRT adds a level of complexity that may cause some challenges during surgery. This was evidenced by the fact that only 19 of the patients $(45 \%)$ underwent resection and only 14 of the patients ( $74 \%$ of the resected 
FIG. 2 Kaplan-Meier overall survival (OS) curve stratified by induction chemotherapy type and stage from the date of surgery.

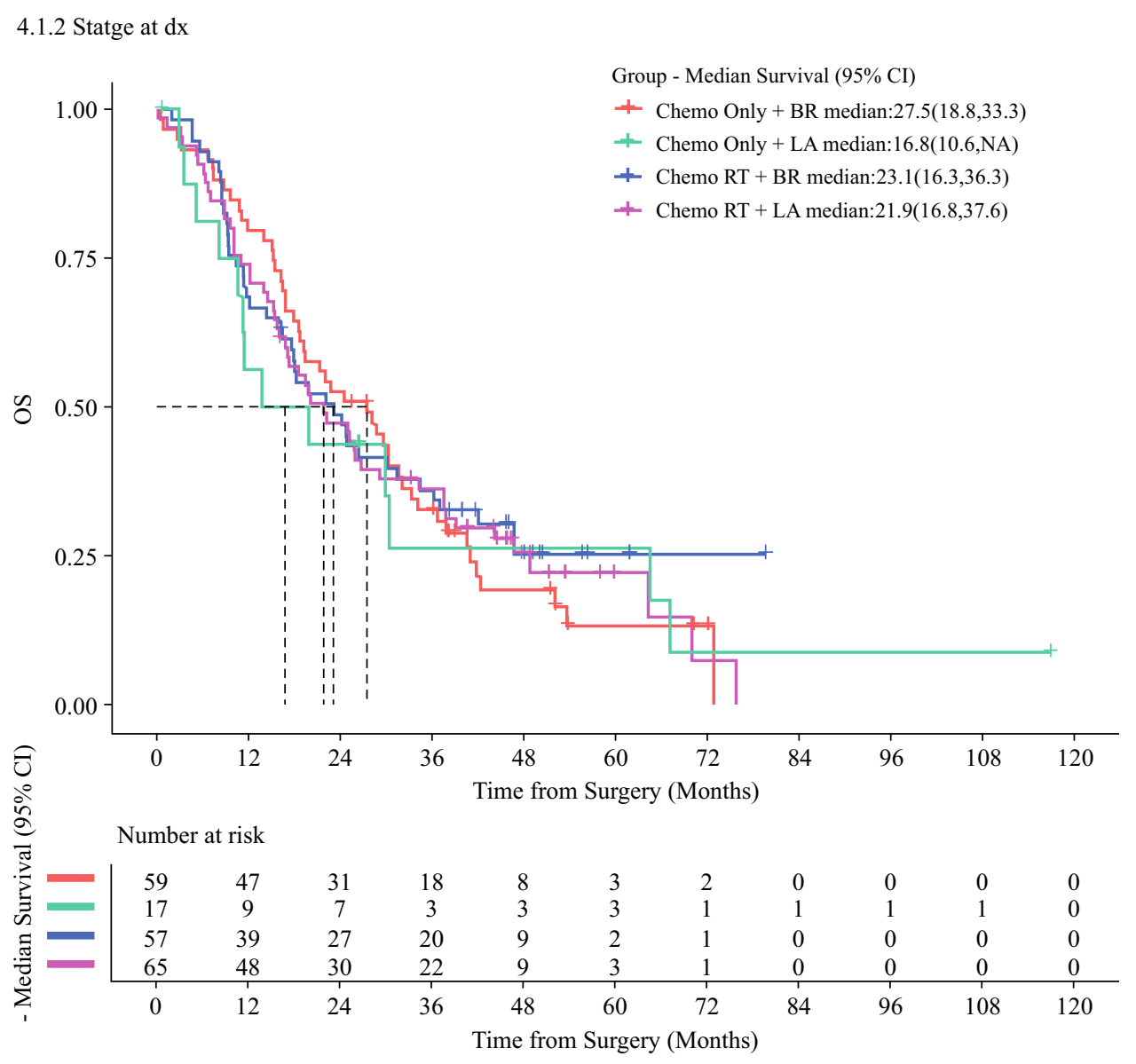

patients; $35 \%$ of the patients treated with radiation therapy) underwent R0 resection. ${ }^{37}$ Nevertheless, pathologic outcomes were improved with SBRT, suggesting that wellselected patients are likely to derive benefit from SBRT and subsequent surgery. ${ }^{37}$

Although the pathologic outcomes, including the R0 resection rate, were significantly improved with SBRT in our study, the survival outcomes did not differ between our cohorts. Although improvements in systemic control certainly will have the greatest impact on survival outcomes, local failure rates are not trivial, as demonstrated by the fact that more than $40 \%$ of the patients in both cohorts experienced local failure as part of the pattern of the first failure. We believe this highlights the need to continue refining and intensifying the manner in which radiation is delivered for patients with BRPC and LAPC. Dose escalation and modification of field design may represent pathways to achieve this goal. Indeed, in the LAPC setting, data suggest improved outcomes if a higher radiation dose can be safely administered, but the radiation tolerance of nearby stomach and bowel structures remains limiting. ${ }^{38,39}$

These aforementioned data have primarily been in the non-operative setting, but such dose escalation may also be applicable in the neoadjuvant setting. Certainly, dose escalation has shown tremendous improvement in local outcomes at other adenocarcinoma sites that are more conducive to safe administration of "ablative" doses, such as lung and liver tumors. ${ }^{40,41}$ As advances in radiation technologies (motion management, enhanced image guidance, and adaptive therapy) have improved the precision of radiation delivery, dose-escalation studies attempting to deliver ablative doses are underway, but novel strategies to allow dose escalation likely will be needed. ${ }^{42-44}$ Furthermore, the radiation target volume itself (tumor margin and coverage of elective nodal regions) remains non-standardized and likely considerably heterogeneous in practice. Although gross disease plus a 3- to 5-mm margin has traditionally been the target for SBRT, in the preoperative setting it is possible that peri-pancreatic perineural tracts and lymphatic channels represent the more important target. $^{45-51}$

Several limitations of this study must be acknowledged, such as the retrospective, single-institution nature of the study and the associated potential for bias. For example, although balanced between cohorts, the utilization of multiagent chemotherapy regimens were nonetheless different, and the study could not fully control for this difference. Notably, the imbalance of LAPC patients 
TABLE 5 90-Day

perioperative toxicity outcomes

\begin{tabular}{|c|c|c|c|c|c|}
\hline \multirow[b]{2}{*}{ Perioperative toxicity } & \multicolumn{2}{|c|}{$\begin{array}{l}\text { Chemotherapy } \\
n \%\end{array}$} & \multicolumn{2}{|c|}{$\begin{array}{l}\text { Chemotherapy and SBRT } \\
n \%\end{array}$} & \multirow{2}{*}{$\begin{array}{l}p \text { Value* } \\
0.71\end{array}$} \\
\hline & & & & & \\
\hline Delayed gastric emptying & 15 & 21.1 & 21 & 17.8 & \\
\hline Postoperative pancreatic fistula & 10 & 14.1 & 11 & 9.3 & \\
\hline Intra-abdominal abscess & 3 & 4.2 & 10 & 8.5 & \\
\hline Bleeding events due to surgery & 2 & 2.8 & 3 & 2.5 & \\
\hline Bleeding event due to SBRT & 0 & 0 & 4 & 3.4 & \\
\hline Bleeding events not due to SBRT/surgery & 0 & 0 & 2 & 1.7 & \\
\hline Wound complications & 16 & 22.5 & 20 & 16.9 & \\
\hline Sepsis & 1 & 1.4 & 7 & 5.9 & \\
\hline Chyle leak & 2 & 2.8 & 9 & 7.6 & \\
\hline Small bowel obstruction & 4 & 5.6 & 3 & 2.5 & \\
\hline Biliary leakage & 0 & 0 & 3 & 2.5 & \\
\hline Duodeno or gastrojejunostomy leak & 0 & 0 & 0 & 0 & \\
\hline Enterocutaneous fistula & 0 & 0 & 0 & 0 & \\
\hline Mesenteric venous thrombosis & 0 & 0 & 1 & 0.8 & \\
\hline Urinary tract infection & 3 & 4.2 & 4 & 3.4 & \\
\hline Clostridium difficile colitis & 2 & 2.8 & 6 & 5.1 & \\
\hline $\mathrm{DVT} / \mathrm{PE}$ & 2 & 2.8 & 4 & 3.4 & \\
\hline Respiratory complications & 3 & 4.2 & 4 & 3.4 & \\
\hline Renal failure & 1 & 1.4 & 1 & 0.8 & \\
\hline Cardiac event & 4 & 5.6 & 5 & 4.2 & \\
\hline Cerebral vascular event & 1 & 1.4 & 0 & 0 & \\
\hline Need for repeat surgery & 3 & 4.2 & 4 & 3.4 & \\
\hline 90-Day mortality & 2 & 2.8 & 2 & 1.7 & 0.62 \\
\hline \multicolumn{6}{|l|}{ Clavien-Dindo classification } \\
\hline No & 30 & 42.3 & 52 & 44.1 & \\
\hline Grade 1 & 12 & 16.9 & 18 & 15.3 & \\
\hline Grade 2 & 16 & 22.5 & 29 & 24.6 & \\
\hline Grade $3 a$ & 8 & 11.3 & 12 & 10.0 & \\
\hline Grade $3 b$ & 3 & 4.2 & 6 & 5.1 & \\
\hline Grade $4 \mathrm{a}$ & 2 & 2.8 & 1 & 0.8 & \\
\hline Grade $4 b$ & 0 & 0.0 & 0 & 0.0 & \\
\hline Grade 5 & 0 & 0.0 & 0 & 0.0 & \\
\hline Total grade $\geq 3$ & 13 & 18.3 & 19 & 15.8 & 0.81 \\
\hline
\end{tabular}

SBRT, stereotactic body radiation therapy; DVT, deep venous thrombosis; PE, pulmonary embolism between treatment arms was more likely to have influenced outcomes negatively in the nCT-SBRT cohort, but it also should be noted that resectability staging (BRPC vs LAPC) may not necessarily be clearly associated with differences in survival outcomes. Additionally, selection bias may have included the decision to administer neoadjuvant radiation to patients with a less robust response to neoadjuvant chemotherapy alone. In addition, patients found to have metastatic disease with restaging after induction therapy before surgery or at the time of surgical exploration would have been excluded from this analysis. Furthermore, we did not have data available to assess the proportion of patients at our institution who received either nCT or nCTSBRT and could not proceed to surgical exploration.

Finally, although the study was focused on pathologic outcomes, clinical outcomes such as differences in local progression-free survival, overall progression-free survival, and ultimately overall survival represent the more important end points. Certainly, exploration of ways in which radiation can be refined to influence such outcomes should be the subject of future study. 


\section{CONCLUSION}

Despite a significantly larger proportion of patients with LAPC, those treated with nCT-SBRT were more likely to have a robust pathologic response and undergo marginnegative resection than those treated with $\mathrm{nCT}$ alone. Although the nCT-SBRT cohort received less adjuvant therapy than the $\mathrm{nCT}$ cohort, and although $30 \%$ of the $\mathrm{nCT}$ patients received adjuvant chemoradiation, the survival outcomes still were similar in both cohorts. More data are needed to refine the determination of which patients benefit from neoadjuvant SBRT and how radiation administration can be further optimized to influence locoregional control, limit toxicity, and improve survival outcomes.

\section{APPENDIX 1}

Patient eligibility and follow-up evaluation. Patients were eligible for inclusion in this study if they had each of the following: a histologic diagnosis of pancreatic adenocarcinoma, a radiographic diagnosis of borderline resectable pancreatic cancer (BRPC) or locally advanced pancreatic cancer (LAPC) per the National Comprehensive Cancer Network $(\mathrm{NCCN})$ guidelines, ${ }^{51}$ neoadjuvant therapy before surgical resection, surgical exploration, and sufficient follow-up evaluation, defined as two or more clinical encounters after surgery. Clinical follow-up evaluation for the patients in the study consisted of a history as well as a physical and pancreatic protocol computed tomography scan, initially obtained at 3-month intervals after surgery, with subsequent intervals determined by the clinical team. Cancer antigen (CA) 19-9 levels also were generally obtained at follow-up visits but at the discretion of the clinical team.

\section{APPENDIX 2}

Stereotactic body radiation therapy (SBRT) planning and treatment objectives. In brief, after placement of fiducials in the pancreatic tumor under endoscopic ultrasound guidance, patients in supine position with arms up underwent a computed tomography simulation with intravenous contrast and immobilization using a Vac-lok (CIVCO Medical Solutions, Coralville, IA, USA) or Alpha-cradle (CIVCO Medical Solutions, Coralville, IA, USA). Patients were treated to a dose of 33 Gy (10-15\% heterogeneity allowed) in five fractions on five consecutive weekdays. Motion management was most commonly addressed using active breathing control (ABC; Elekta, Stockholm, Sweden), although a minority of patients were treated under a free-breathing approach using a customized internal tumor volume expansion based on assessment with a four-dimensional computed tomography scan. The target volume included gross disease as well as the full circumference of the involved vasculature at the level of involvement. Planning tumor volume (PTV) margin expansion was 2 to $3 \mathrm{~mm}$. After SBRT, the patients in both cohorts received further systemic therapy before and after surgery at the discretion of the medical oncologist. A subset of patients in the neoadjuvant chemotherapy (nCT) cohort with positive margins after surgery received adjuvant radiation with conventional fractionation (25-30 fractions, often with concurrent capecitabine).

\section{APPENDIX 3}

Statistical analysis. Overall survival was recorded as the time from the end of surgery to death. The date of death was sourced from medical records and the Social Security Death Index. If the date of death was not available, survival was censored at the date of the last recorded clinical encounter. Progression-free survival (PFS) was measured as the interval from the end of surgery to the time of the first radiographic evidence of failure or death, whichever occurred earlier, and censored at the date of the last recorded imaging follow-up evaluation. Local progressionfree survival (LPFS) and distant metastasis-free survival (DMFS) were recorded as the time for the first occurrence of locoregional or distant failure, respectively, or death, whichever occurred earlier. Overall survival (OS), progression free-survival (PFS), local PFS (LPFS), and distant metastasis-free survival (DMFS) were estimated using Kaplan-Meier analysis and compared with the log-rank test. Clinical and treatment covariates were compared between the two patient cohorts using Fisher's exact test or Student's $t$ test. The Cox regression model was used to estimate the hazard ratio (HR) between the two cohorts as well as other prognostic factors, with the corresponding 95\% confidence interval (CI). The inverse probability of treatment weighting (IPTW) using the propensity score was applied to address confounders in comparing the cohorts for pathologic and survival outcomes and assessing the effect of adjuvant chemotherapy within each cohort. Age at diagnosis, sex, National Comprehensive Cancer Network (NCCN) stage at diagnosis, tumor location, pre- and postneoadjuvant chemotherapy CA19-9, baseline Eastern Cooperative Oncology Group (ECOG), and duration and type of neoadjuvant chemotherapy in each cohort were used to calculate the propensity score using generalized boosted regression model. ${ }^{52}$ Cancer antigen 19-9 (CA 19-9) measurements and ECOG were not available for all the patients. Multiple imputation by chained equations (MICE) was used to account for missing data. ${ }^{53}$ All the clinical and treatment covariates presented in Table 1 were 
included in the multiple imputation model. Average treatment effect (ATE) and average treatment effect on treated (ATT) were estimated using logistic regression or Cox regression (for survival outcomes) with the IPTW in each of 20 imputed datasets and then pooled using Rubin's rules. ${ }^{54,55}$ A two-sided significance level of 0.05 was used throughout. Statistical analyses were performed using $\mathrm{R}$ 4.0.1. (R Foundation for Statistical Computing, Vienna, Austria.) $)^{56,57}$

Supplementary Information The online version contains supplementary material available at https://doi.org/10.1245/s10434021-11202-8.

ACKNOWLEDGMENT This study received funding from the Skip Viragh Center for Pancreatic Cancer Research and Clinical Care and McKnight Family Foundation.

OPEN ACCESS This article is licensed under a Creative Commons Attribution 4.0 International License, which permits use, sharing, adaptation, distribution and reproduction in any medium or format, as long as you give appropriate credit to the original author(s) and the source, provide a link to the Creative Commons licence, and indicate if changes were made. The images or other third party material in this article are included in the article's Creative Commons licence, unless indicated otherwise in a credit line to the material. If material is not included in the article's Creative Commons licence and your intended use is not permitted by statutory regulation or exceeds the permitted use, you will need to obtain permission directly from the copyright holder. To view a copy of this licence, visit http://creativecommons. org/licenses/by/4.0/.

DISCLOSURE Chen Hu received personal fees from Merck \& Co. Dung Le serves on advisory boards for Merck, Bristol Myers Squibb, and Janssen and has received research funding from Merck, Bristol Myers Squibb, Aduro Biotech, Curegenix, Medivir, and Nouscom. She has received speaking honoraria from Merck and is an inventor of licensed intellectual property related to technology for mismatch repair deficiency for diagnosis and therapy (WO2016077553A1) from Johns Hopkins University. The terms of these arrangements are being managed by Johns Hopkins. Joseph Herman has research funding from the Canopy Cancer Collective and has been a consultant for Boston Scientific.

\section{REFERENCES}

1. Siegel RL, Miller KD, Fuchs HE, Jemal A. Cancer statistics, 2021. CA Cancer J Clin. 2021;71:7-33. https://doi.org/10.3322/ caac.21654 (Epub 12 January 2021 PMID: 33433946).

2. Rahib L, Smith BD, Aizenberg R, Rosenzweig AB, Fleshman JM, Matrisian LM. Projecting cancer incidence and deaths to 2030: the unexpected burden of thyroid, liver, and pancreas cancers in the United States. Cancer Res. 2014;74:2913-21.

3. Gemenetzis G, Groot VP, Blair AB, et al. Survival in locally advanced pancreatic cancer after neoadjuvant therapy and surgical resection. Ann Surg. 2019;270:340-7. https://doi.org/10. 1097/SLA.0000000000002753.

4. Ferrone CR, Marchegiani G, Hong TS, Ryan DP, Deshpande V, McDonnell EI, et al. Radiological and surgical implications of neoadjuvant treatment with FOLFIRINOX for locally advanced and borderline resectable pancreatic cancer. Ann Surg. 2015;261:12-7. https://doi.org/10.1097/SLA.0000000000000 867.

5. Faris JE, Blaszkowsky LS, McDermott S, et al. FOLFIRINOX in locally advanced pancreatic cancer: the Massachusetts General Hospital Cancer Center experience. Oncologist. 2013;18:543-8. h ttps://doi.org/10.1634/theoncologist.2012-0435.

6. Javed AA, Wright MJ, Siddique A, Blair AB, Ding D, Burkhart $\mathrm{RA}$, et al. Outcome of patients with borderline resectable pancreatic cancer in the contemporary era of neoadjuvant chemotherapy. J Gastrointest Surg. 2019;23:112-21. https://doi. org/10.1007/s11605-018-3966-8 (Epub 21 September 2018. PMID: 30242644; PMCID: PMC6329638).

7. Murphy JE, Wo JY, Ryan DP, et al. Total neoadjuvant therapy with FOLFIRINOX followed by individualized chemoradiotherapy for borderline resectable pancreatic adenocarcinoma: a phase 2 clinical trial [published correction appears in JAMA Oncol. 2018;4:1439]. JAMA Oncol. 2018;4:963-9. https://doi.org/10.10 01/jamaoncol.2018.0329.

8. Janssen QP, Buettner S, Suker M, et al. Neoadjuvant FOLFIRINOX in patients with borderline resectable pancreatic cancer: a systematic review and patient-level meta-analysis. J Natl Cancer Inst. 2019;111:782-94. https://doi.org/10.1093/jnci/djz073.

9. Conroy T, Hammel P, Hebbar M, Ben Abdelghani M, Wei AC, Raoul JL, et al. Canadian Cancer Trials Group and the UnicancerGI-PRODIGE Group. FOLFIRINOX or gemcitabine as adjuvant therapy for pancreatic cancer. $N$ Engl $J$ Med. 2018;379:2395-406. https://doi.org/10.1056/NEJMoa1809775 (PubMed PMID: 30575490).

10. Von Hoff DD, Ervin T, Arena FP, Chiorean EG, Infante J, Moore $\mathrm{M}$, et al. Increased survival in pancreatic cancer with nab-paclitaxel plus gemcitabine. N Engl J Med. 2013;369:1691-703. h ttps://doi.org/10.1056/NEJMoa1304369 (Epub 16 October 2013. PMID: 24131140; PMCID: PMC4631139).

11. Perri G, Prakash LR, Katz MHG. Response to preoperative therapy in localized pancreatic cancer. Front Oncol. 2020;10:516. https://doi.org/10.3389/fonc.2020.00516.PMID:32351893;PMCI D:PMC7174698.

12. Herman JM, Chang DT, Goodman KA, et al. Phase 2 multiinstitutional trial evaluating gemcitabine and stereotactic body radiotherapy for patients with locally advanced unresectable pancreatic adenocarcinoma. Cancer. 2015;121:1128-37. https://doi.org/10.1002/cncr.29161.

13. Chang DT, Schellenberg D, Shen J, et al. Stereotactic radiotherapy for unresectable adenocarcinoma of the pancreas. Cancer. 2009;115:665-72. https://doi.org/10.1002/cncr.24059.

14. Wild AT, Herman JM, Dholakia AS, Moningi S, Lu Y, Rosati LM, et al. Lymphocyte-sparing effect of stereotactic body radiation therapy in patients with unresectable pancreatic cancer. Int J Radiat Oncol Biol Phys. 2016;94:571-9. https://doi.org/10.10 16/j.ijrobp.2015.11.026 (Epub 1 December 2015. PMID: 26867885; PMCID: PMC4847529).

15. Park JJ, Hajj C, Reyngold M, Shi W, Zhang Z, Cuaron JJ. Stereotactic body radiation vs intensity-modulated radiation for unresectable pancreatic cancer. Acta Oncologica. 2017;56(12):1746-53. https://doi.org/10.1080/0284186X.2017. 1342863.

16. Rao AD, Sugar EA, Chang DT, Goodman KA, Hacker-Prietz A, Rosati LM, et al. Patient-reported outcomes of a multicenter phase 2 study investigating gemcitabine and stereotactic body radiation therapy in locally advanced pancreatic cancer. Pract Radiat Oncol. 2016;6:417-24. https://doi.org/10.1016/j.prro.201 6.05.005 (Epub 25 May 2016. PMID: 27552809; PMCID: PMC5572652).

17. Jang JY, Han Y, Lee H, Kim SW, Kwon W, Lee KH, et al. Oncological benefits of neoadjuvant chemoradiation with 
gemcitabine versus upfront surgery in patients with borderline resectable pancreatic cancer: a prospective, randomized, openlabel, multicenter phase 2/3 trial. Ann Surg. 2018;268:215-22. h ttps://doi.org/10.1097/SLA.0000000000002705

(PMID: 29462005).

18. Versteijne E, Suker M, Groothuis K, Akkermans-Vogelaar JM, Besselink MG, Bonsing BA, et al. Dutch Pancreatic Cancer Group. Preoperative chemoradiotherapy versus immediate surgery for resectable and borderline resectable pancreatic cancer: results of the dutch randomized phase III PREOPANC trial. $J$ Clin Oncol. 2020;38:1763-73. https://doi.org/10.1200/JCO.19.0 2274 (Epub 27 February 2020. PMID: 32105518).

19. Hammel P, Huguet F, van Laethem JL, Goldstein D, Glimelius B, Artru P, et al. LAP07 Trial Group. Effect of chemoradiotherapy vs chemotherapy on survival in patients with locally advanced pancreatic cancer controlled after 4 months of gemcitabine with or without erlotinib: the LAP07 randomized clinical trial. JAMA. 2016;315(17):1844-53. https://doi.org/10.1001/jama.2016.4324. PMID: 27139057

20. Washington MK, Berlin J, Branton P, Burgart LJ, Carter DK, Fitzgibbons PL, et al. Members of the Cancer Committee, College of American Pathologists. Protocol for the examination of specimens from patients with primary carcinoma of the colon and rectum. Arch Pathol Lab Med. 2009;133(10):1539-51. https://d oi.org/10.5858/133.10.1539. PMID: 19792043; PMCID: PMC2901838

21. Dindo D, Demartines N, Clavien PA. Classification of surgical complications: a new proposal with evaluation in a cohort of 6336 patients and results of a survey. Ann Surg. 2004;240:205-13.

22. Kalser MH, Ellenberg SS. Pancreatic cancer: adjuvant combined radiation and chemotherapy following curative resection. Arch Surg. 1985;120:899-903. https://doi.org/10.1001/archsurg.1985. 01390320023003 .

23. Chauffert B, Mornex F, Bonnetain F, Rougier P, Mariette C, Bouche $\mathrm{O}$, et al. Phase III trial comparing intensive induction chemoradiotherapy (60 Gy, infusional 5-FU and intermittent cisplatin) followed by maintenance gemcitabine with gemcitabine alone for locally advanced unresectable pancreatic cancer: definitive results of the 2000-01 FFCD/SFRO study. Ann Oncol. 2008;19:1592-9. https://doi.org/10.1093/annonc/mdn281.

24. Klaassen DJ, MacIntyre JM, Catton GE, et al. Treatment of locally unresectable cancer of the stomach and pancreas: a randomized comparison of 5 -fluorouracil alone with radiation plus concurrent and maintenance 5 -fluorouracil-an eastern cooperative oncology group study. J Clin Oncol. 1985;3:373-8. https://doi. org/10.1200/JCO.1985.3.3.373.

25. Loehrer PJ Sr, Feng Y, Cardenes H, Wagner L, Brell JM, Cella D, et al. Gemcitabine alone versus gemcitabine plus radiotherapy in patients with locally advanced pancreatic cancer: an Eastern Cooperative Oncology Group trial. J Clin Oncol. 2011;29:4105-12. https://doi.org/10.1200/JCO.2011.34.8904.

26. Lee J, Lee JC, Gromski MA, et al. Clinical outcomes of FOLFIRINOX in locally advanced pancreatic cancer: a single-center experience. Medicine Baltimore. 2018;97:e13592. https://doi.org/ 10.1097/MD.0000000000013592.

27. Khushman M, Dempsey N, Maldonado JC, Loaiza-Bonilla A, Velez M, Carcas L. Full-dose neoadjuvant FOLFIRINOX is associated with prolonged survival in patients with locally advanced pancreatic adenocarcinoma. Pancreatology. 2015;15:667-73. https://doi.org/10.1016/j.pan.2015.08.010 (ISSN 1424-3903).

28. Hackert T, Sachsenmaier M, Hinz U, Schneider L, Michalski CW, Springfeld C, et al. Locally advanced pancreatic cancer. Ann Surg. 2016;264:457-63. https://doi.org/10.1097/SLA.000000000 0001850 .
29. Matsumoto I, Kamei K, Omae K, Suzuki S, Matsuoka H, Mizuno $\mathrm{N}$, et al. FOLFIRINOX for locally advanced pancreatic cancer: results and prognostic factors of subset analysis from a nationwide multicenter observational study in Japan. Pancreatology. 2019;19:296-301. https://doi.org/10.1016/j.pan.2019.01.001 (Epub 4 January 2019 PMID: 30638853).

30. Mellon EA, Hoffe SE, Springett GM, Frakes JM, Strom TJ, Hodul PJ, et al. Long-term outcomes of induction chemotherapy and neoadjuvant stereotactic body radiotherapy for borderline resectable and locally advanced pancreatic adenocarcinoma. Acta Oncol. 2015;54:979-85. https://doi.org/10.3109/0284186X.2015. 1004367 (Epub 3 March 2015 PMID: 25734581).

31. Toesca DAS F, Ahmed F, Kashyap M, Baclay JRM, von Eyben R, Pollom EL, et al. Intensified systemic therapy and stereotactic ablative radiotherapy dose for patients with unresectable pancreatic adenocarcinoma. Radiother Oncol. 2020;152:63-9. https://d oi.org/10.1016/j.radonc.2020.07.053 (Epub 5 August 2020. PMID: 32763253).

32. Murphy JE, Wo JY, Ryan DP, et al. Total neoadjuvant therapy with FOLFIRINOX in combination with losartan followed by chemoradiotherapy for locally advanced pancreatic cancer: a phase 2 clinical trial. JAMA Oncol. 2019;5:1020-7. https://doi. org/10.1001/jamaoncol.2019.0892.

33. Garnier J, Ewald J, Marchese U, et al. Outcomes of patients with initially locally advanced pancreatic adenocarcinoma who did not benefit from resection: a prospective cohort study. BMC Cancer. 2020;20:203. https://doi.org/10.1186/s12885-020-6690-1.

34. Suker M, Nuyttens JJ, Eskens FALM, Haberkorn BCM, Coene P-PLO, van der Harst E. Efficacy and feasibility of stereotactic radiotherapy after FOLFIRINOX in patients with locally advanced pancreatic cancer (LAPC-1 trial). Eclinical Med. 2019;17:100200. https://doi.org/10.1016/j.eclinm.2019.10.013 (ISSN 2589-5370).

35. Teriaca MA, Loi M, Suker M, Eskens FALM, van Eijck CHJ, Nuyttens JJ. A phase II study of stereotactic radiotherapy after FOLFIRINOX for locally advanced pancreatic cancer (LAPC-1 trial): long-term outcome. Radiother Oncol. 2021;155:232-6. h ttps://doi.org/10.1016/j.radonc.2020.11.006 (ISSN 0167-8140).

36. Katz MH, Shi Q, Ahmad SA, Herman JM, Marsh Rde W, Collisson E, et al. Preoperative modified FOLFIRINOX treatment followed by capecitabine-based chemoradiation for borderline resectable pancreatic cancer: Alliance for Clinical Trials in Oncology Trial A021101. JAMA Surg. 2016;151:e161137. http s://doi.org/10.1001/jamasurg.2016.1137 (Epub 17 August 2016. PMID: 27275632; PMCID: PMC5210022).

37. Katz MHG, Ou FS, Herman JM, Ahmad SA, Wolpin B, Marsh R, et al. Alliance for Clinical Trials in Oncology (ALLIANCE) trial A021501: preoperative extended chemotherapy vs. chemotherapy plus hypofractionated radiation therapy for borderline resectable adenocarcinoma of the head of the pancreas. BMC Cancer. 2017;17:505. https://doi.org/10.1186/s12885-017-3441$\mathrm{z}$.

38. Sunil K, Awalpreet C, Yelin S, Hsiang-Chun C, Arvind R, Prajnan $\mathrm{D}$, et al. Focal radiation therapy dose escalation improves overall survival in locally advanced pancreatic cancer patients receiving induction chemotherapy and consolidative chemoradiation. Int J Radiat Oncol Biol Phys. 2016;94:755-65. https://doi. org/10.1016/j.ijrobp.2015.12.003.

39. Reyngold M, O'Reilly EM, Varghese AM, Fiasconaro M, Zinovoy $M$, Romesser PB, et al. Association of ablative radiation therapy with survival among patients with inoperable pancreatic cancer. JAMA Oncol. 2021;7(5):735-8. https://doi.org/10.1001/ja maoncol.2021.0057. PMID: 33704353; PMCID: PMC7953335

40. Timmerman R, Paulus R, Galvin J, et al. Stereotactic body radiation therapy for inoperable early stage lung cancer. JAMA. 2010;303:1070-6. https://doi.org/10.1001/jama.2010.261. 
41. Rusthoven KE, Kavanagh BD, Cardenes H, Stieber VW, Burri SH, Feigenberg SJ, et al. Multi-institutional phase I/II trial of stereotactic body radiation therapy for liver metastases. J Clin Oncol. 2009;27:1572-8. https://doi.org/10.1200/JCO.2008.19.63 29 (Epub 2 March 2009 PMID: 19255321)

42. Rudra S, Jiang N, Rosenberg SA, Olsen JR, Roach MC, Wan L, et al. Using adaptive magnetic resonance image-guided radiation therapy for treatment of inoperable pancreatic cancer. Cancer Med. 2019;8:2123-32. https://doi.org/10.1002/cam4.2100 (Epub 1 April 2019. PMID: 30932367; PMCID: PMC6536981).

43. Rao AD, Feng Z, Shin EJ, He J, Waters KM, Coquia S, et al. A novel absorbable radiopaque hydrogel spacer to separate the head of the pancreas and duodenum in radiation therapy for pancreatic cancer. Int J Radiat Oncol Biol Phys. 2017;99:1111-20. https://d oi.org/10.1016/j.ijrobp.2017.08.006 (Epub 14 August 2017. PMID: 28943075; PMCID: PMC5699940).

44. Keane FK, Wo JY, Ferrone CR, Clark JW, Blaszkowsky LS, Allen JN, et al. Intraoperative radiotherapy in the era of intensive neoadjuvant chemotherapy and chemoradiotherapy for pancreatic adenocarcinoma. Am J Clin Oncol. 2018;41:607-12.

45. Levy MJ, Topazian M, Keeney G, Clain JE, Gleeson F, Rajan E, et al. Preoperative diagnosis of extrapancreatic neural invasion in pancreatic cancer. Clin Gastroenterol Hepatol. 2006;4:1479-82. https://doi.org/10.1016/j.cgh.2006.08.012 (Epub 13 November 2006 PMID: 17101297).

46. Nakao A, Harada A, Nonami T, Kaneko T, Takagi H. Clinical significance of carcinoma invasion of the extrapancreatic nerve plexus in pancreatic cancer. Pancreas. 1996;12:357-61. https://d oi.org/10.1097/00006676-199605000-00006 (PMID: 8740402).

47. Tian H, Mori H, Matsumoto S, Yamada Y, Kiyosue H, Ohta M, Kitano S. Extrapancreatic neural plexus invasion by carcinomas of the pancreatic head region: evaluation using thin-section helical CT. Radiat Med. 2007;25:141-7. https://doi.org/10.1007/ s11604-006-0115-1 (Epub 28 May 2007 PMID: 17514364).

48. Rhieu BH, Han-Oh S, Guss ZD, Cheng Z, Meyer JJ, Herman JM, Narang A. Patterns of local failure after neoadjuvant radiation for borderline resectable and locally advanced pancreatic adenocarcinoma. Int J Radiat Oncol Biol Phys. 2019;105:E243. https://doi. org/10.1016/j.ijrobp.2019.06.1924.
49. Schneider M, Strobel O, Hackert T, Büchler MW. Pancreatic resection for cancer: the Heidelberg technique. Langenbecks Arch Surg. 2019;404:1017-22. https://doi.org/10.1007/s00423-019-01 839-1 (Epub 14 November 2019 PMID: 31728630).

50. Hackert T, Strobel O, Michalski CW, Mihaljevic AL, Mehrabi A, Müller-Stich B, et al. The TRIANGLE operation: radical surgery after neoadjuvant treatment for advanced pancreatic cancer: a single-arm observational study. HPB Oxford. 2017;19:1001-7. h ttps://doi.org/10.1016/j.hpb.2017.07.007 (Epub 31 August 2017 PMID: 28838632).

51. Nagakawa Y, Yi SQ, Takishita C, Sahara Y, Osakabe H, Kiya Y, et al. Precise anatomical resection based on structures of nerve and fibrous tissue around the superior mesenteric artery for mesopancreas dissection in pancreaticoduodenectomy for pancreatic cancer. J Hepatobiliary Pancreat Sci. 2020;27:342-51. h ttps://doi.org/10.1002/jhbp.725 (Epub 11 March 2020 PMID: 32048456).

52. National Comprehensive Cancer Network. Pancreatic Adenocarcinoma (version 1.2021). Retrieved 24 November 2020 at h ttps://www.nccn.org/professionals/physician_gls/pdf/pancreatic.p df.

53. Friedman J, Hastie T, Tibshirani R. Additive logistic regression: a statistical view of boosting (with discussion and a rejoinder by the authors). Anna Stats. 2000;28:337-407.

54. van Buuren S, Brand JPL, Groothuis-Oudshoorn CGM, Rubin DB. Fully conditional specification in multivariate imputation. $J$ Stat Comput Simulat. 2006;76(12):1049-64.

55. Austin PC. An introduction to propensity score methods for reducing the effects of confounding in observational studies. Multivariate Behav Res. 2011;46:399-424.

56. Barnard J, Rubin DB. Small-sample degrees of freedom with multiple imputation. Biometrika. 1999;86:948-55.

57. $\mathrm{R}$ Core Team. R: a language and environment for statistical computing. Vienna: R Foundation for Statistical Computing; 2021.

Publisher's Note Springer Nature remains neutral with regard to jurisdictional claims in published maps and institutional affiliations. 